\title{
Target sequence heterogeneity causes the 'hook effect' in fluorescent dye-based quantitative PCR
}

Kristina Warton*,1,2 iD, Yue $\mathbf{X u}^{3}$ iD \& Caroline Elizabeth Ford ${ }^{1}$ iD

${ }^{1}$ School of Women's \& Children's Health, University of New South Wales, Sydney, NSW, Australia; ${ }^{2}$ Garvan Institute of Medical Research, Genomics \& Epigenetics Division, Darlinghurst, NSW 2010, Australia; ${ }^{3}$ School of Medical Sciences, University of New South Wales, Sydney, NSW, Australia; *Author for correspondence: k.warton@unsw.edu.au

BioTechniques 69: 81-83 (August 2020) 10.2144/btn-2020-0016

First draft submitted: 12 February 2020; Accepted for publication: 14 April 2020; Published online: 5 June 2020

\section{ABSTRACT}

The 'hook effect' describes a phenomenon in quantitative PCR (qPCR) amplification curves where fluorescence values decrease following an initial amplification phase. We propose that in intercalating dye-based qPCR, the 'hook effect' is due to the amplification of heterogeneous but related DNA targets. The decrease in fluorescence at later cycles occurs because the related products self-anneal to form a DNA heteroduplex with a melt temperature below the temperature at which the fluorescence measurement is made. We show this experimentally using qPCR of Alu family repetitive DNA elements.

KEYWORDS:

hook effect $\bullet$ optimization $\bullet$ quantitation $\bullet$ quantitation artifact $\bullet$ quantitative PCR

The 'hook effect' in quantitative PCR (qPCR) describes a phenomenon in DNA amplification curves where, following exponential increase, fluorescence values decrease rather than maintaining a plateau [1,2]. Where hybridization probes are used, the decrease is proposed to be due to high concentrations of PCR amplicons at later cycles self-annealing with greater efficiency than amplicon-probe complexes [2]. Incorrect baseline correction can also cause an apparent decrease in fluorescence in early or late cycles, regardless of PCR chemistry [3]. However, for reactions without baseline artifacts, the mechanistic basis of the 'hook effect' in qPCR with intercalating dye has not been described.

We postulated that in qPCR with intercalating dye, the 'hook effect' can occur if the target sequence is heterogeneous and the melt temperature of the amplified DNA heteroduplex is below the temperature at which the fluorescence reading is acquired.

We tested this model using PCR primers that target Alu sequences [4]. Alu sequences are a family of repetitive DNA elements found in primate genomes at around $1 \times 10^{6}$ copies per genome [5]. The sequences are related but not identical, creating a heterogeneous target for PCR.

Alu primers for a 115-base pair product were as designed by Umetani and colleagues [4], and amplification conditions were as we previously described [6]. However, modified extension temperatures were used to achieve a fluorescence capture step that was below the melt temperature of the perfectly matched PCR product but above the melt temperature of the PCR product heteroduplex, which we postulated would create the 'hook effect'. In order to compare this with standard PCR conditions, a gradient PCR machine was used to create a three-step cycling profile with a $95^{\circ} \mathrm{C}$ DNA dissociation step and a $60^{\circ} \mathrm{C}$ primer annealing step, followed by either a $72^{\circ} \mathrm{C}$ extension step or a $77^{\circ} \mathrm{C}$ extension step in the same run. The two different extension temperatures were incorporated into the PCR run by applying the temperature gradient to the extension step rather than the primer annealing step, such that the samples underwent either $72^{\circ} \mathrm{C}$ extension or $77^{\circ} \mathrm{C}$ extension. Seventy-two degree was chosen as the standard PCR extension temperature, while $77^{\circ} \mathrm{C}$ was chosen because, from our previous observations of the Alu 115-base pair PCR reaction, this fell just above the melt peak derived from dissociation of the postulated DNA heteroduplexes. Fluorescence was read at the end of the extension step for each cycle, and a melt analysis from 60 to $95^{\circ} \mathrm{C}$ was carried out at the end of the amplification. We anticipated that if the 'hook effect' is indeed created by fluorescence measurement above the melt temperature of the DNA heteroduplex, it would occur at the $77^{\circ} \mathrm{C}$, but not at the $72^{\circ} \mathrm{C}$, extension temperature.

A sigmoidal amplification curve typical of qPCR was observed when fluorescence was measured at the end of the $72^{\circ} \mathrm{C}$ extension step; however, fluorescence measurement at the end of the $77^{\circ} \mathrm{C}$ extension step resulted in a 'hook effect' amplification curve, as predicted (Figure 1A).

The melt curve from both types of reaction shows two peaks, one with a melt temperature of $88^{\circ} \mathrm{C}$ (peak 1 ), and a second broad peak with a maximum at $76^{\circ} \mathrm{C}$ (peak 2) (Figure 1B). Under our model, peak 2 consists of heteroduplexes made up of self-annealed PCR product, while peak 1 consists of homoduplexes that were either synthesized in the cycle immediately preceding the melt step, 
(A) Amplification

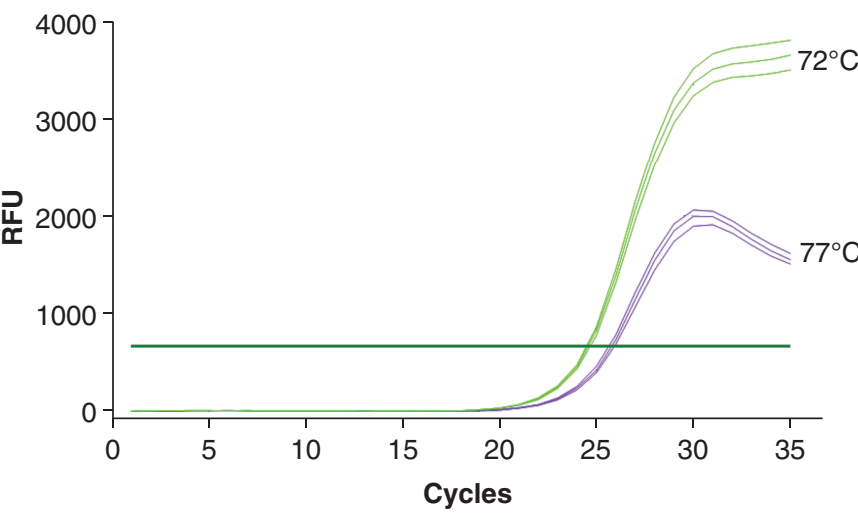

(B) Melt peak

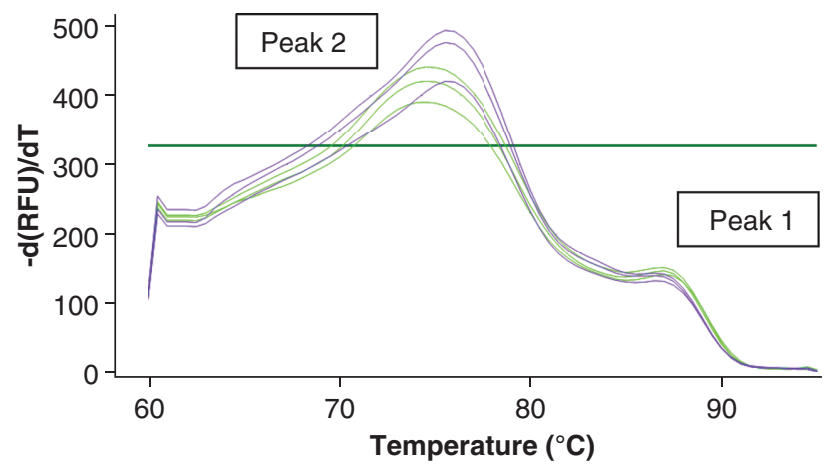

Figure 1. 'Hook effect' created by fluorescence acquisition above the DNA heteroduplex melt temperature. (A) Fluorescence acquisition below the heteroduplex melt temperature $\left(72^{\circ} \mathrm{C}\right.$, green trace) shows a plateau after amplification; fluorescence acquisition above the heteroduplex melt temperature shows the 'hook effect' $\left(77^{\circ} \mathrm{C}\right.$, purple trace). (B) Melt curves for the amplification shown in (A). The cycling data with a log scale $y$-axis is shown in Supplementary Figure 1.

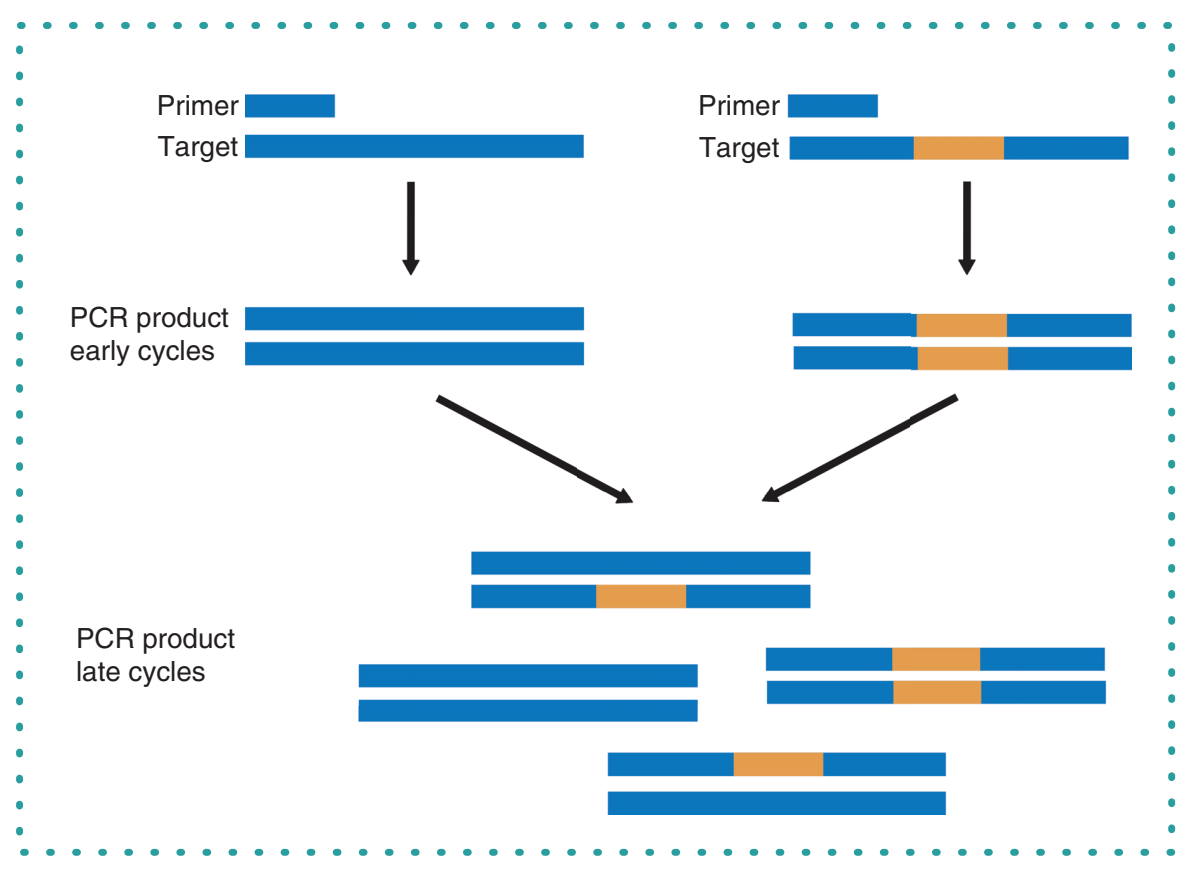

Figure 2. PCR reactions that amplify a heterogeneous target generate a perfectly matched DNA duplex that is the result of primer extension in early cycles of the reaction, and a mismatched DNA duplex that is the result of product self-annealing in late cycles of the reaction.

or represent particularly abundant Alu sequence variants that are present at high concentration in the PCR product and form a perfect match when they self-anneal.

Furthermore, in early PCR cycles, where the ratio of primer to DNA is high, the extension step finishes with a perfectly matched DNA duplex which is the result of strand copying and which has a relatively high melting temperature because the sequence is $100 \%$ complementary. In later cycles, in which the PCR product concentration is high, amplified product self-annealing competes with primer annealing to create heteroduplexes that prevent primer binding and extension. If the heteroduplex has a melting temperature below the extension temperature, it will dissociate during the time the fluorescence is measured, resulting in a fluorescence decrease compared with previous cycles (Figure 2). With progressive cycling, the balance of DNA duplexes shifts further away from newly synthesized product toward self-annealed product, and the fluorescence will decrease further. 
- A Amplification

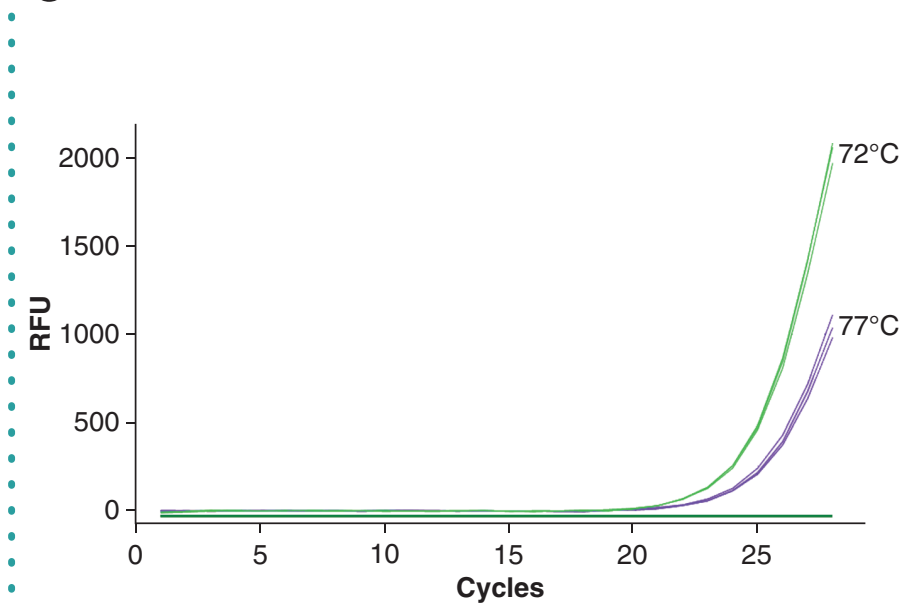

(B) Melt peak

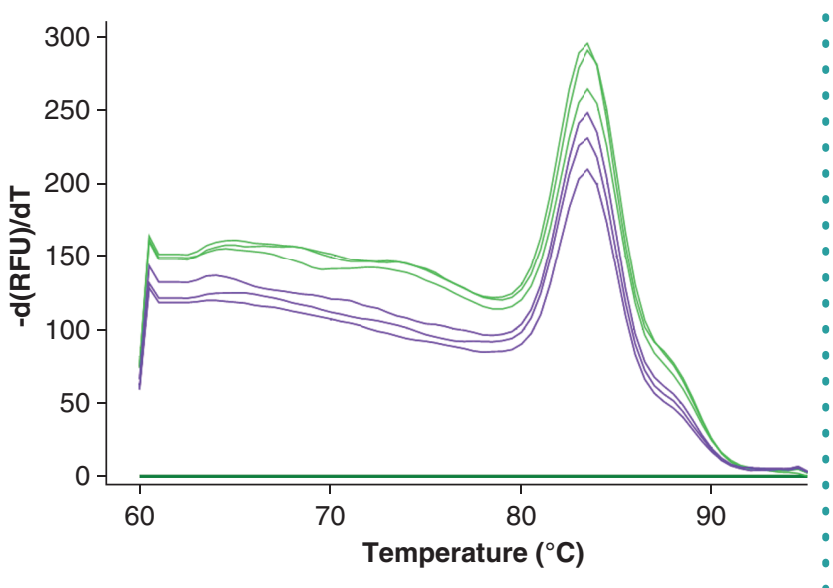

Figure 3. Low cycle number PCR and melt curve of DNA homoduplex resulting from primer extension. (A) Amplification after 28 cycles of PCR with fluorescence acquisition at $72^{\circ} \mathrm{C}$ (green trace) or $77^{\circ} \mathrm{C}$ (purple trace). (B) Melt curves for the PCR run in (A). The curves show only a single peak with a temperature above $80^{\circ} \mathrm{C}$.

We tested this second hypothesis by repeating the PCR reaction with only 28 cycles, as we determined from Figure $1 \mathrm{~A}$ that at 28 cycles significant product self-annealing is not yet taking place. Thus at this point, we would expect only a single melt peak to be present; according to our hypothesis, this would be derived from the PCR product made when the primer was extended just prior to the melt step (Figure 2, early cycles). As predicted, carrying out the melt analysis after 28 cycles of amplification resulted in a single melt peak at around $84^{\circ} \mathrm{C}$ (Figure $\left.3 \mathrm{~A} \& \mathrm{~B}\right)$.

Our data support the hypothesis that a heterogeneous PCR target can generate the 'hook effect' in intercalating dye PCR. Empirically establishing the extent of the heteroduplex mismatches required to produce this effect is beyond the scope of this study; however, singlebase mismatches are clearly insufficient to shift the PCR product's melt temperature significantly. We suggest that the effect requires a small insertion/deletion, or mismatch at multiple sites (such as in repetitive elements or in heterogeneously methylated DNA following bisulfite conversion). In either case, the 'hook effect' should alert the investigator to the possibility that heterogeneous PCR products have been amplified.

While in hybridization probe-based qPCR, the 'hook effect' does not affect efficiency, specificity or target detection [2], this is not the case for intercalating dye-based qPCR, particularly if the validity of the assay requires amplification of a single homogenous target. In that scenario, ignoring the 'hook effect' will lead to incorrect data and conclusions.

\section{Supplementary data}

To view the supplementary data that accompany this paper please visit the journal website at: www.futurescience.com/doi/suppl/10.2144/btn-2020-0016

\section{Author contributions}

All authors were involved in experiment design, interpretation and manuscript preparation. Y Xu carried out the laboratory experiments.

\section{Acknowledgments}

We would like to thank L Hesson of the Garvan Institute of Medical Research for critical reading of the manuscript and feedback.

\section{Financial \& competing interests disclosure}

K Warton is supported by a grant from the Ovarian Cancer Research Foundation (Australia). K Warton holds stock in Guardant Health, Exact Sciences and Epigenomics AG. The authors have no other relevant affiliations or financial involvement with any organization or entity with a financial interest in or financial conflict with the subject matter or materials discussed in the manuscript apart from those disclosed.

No writing assistance was utilized in the production of this manuscript. 


\section{Open access}

This work is licensed under the Attribution-NonCommercial-NoDerivatives 4.0 Unported License. To view a copy of this license, visit http://creativecommons.org/licenses/by-nc-nd/4.0/

\section{References}

1 Burdukiewicz M, Spiess AN, Blagodatskikh KA, Lehmann W, Schierack P, Rodiger S. Algorithms for automated detection of hook effect-bearing amplification curves. Biomol. Detect. Quantif. 16, 1-4 (2018).

2 Keer J. Quantitative real-time PCR analysis. In: Essentials of Nucleic Acid Analysis: A Robust Approach. Birch L, Keer JT (Eds). Cambridge, UK (2008).

3 Nolan T, Hands RE, Bustin SA. Quantification of mRNA using real-time RT-PCR. Nat. Protoc. 1(3), 1559-1582 doi: 1510.1038/nprot.2006.1236 (2006) (Epub ahead of print).

4 Umetani N, Kim J, Hiramatsu S et al. Increased integrity of free circulating DNA in sera of patients with colorectal or periampullary cancer: direct quantitative PCR for ALU repeats. Clin. Chem. 52(6), 1062-1069 (2006).

5 Daniel C, Behm M, Ohman M. The role of Alu elements in the cis-regulation of RNA processing. Cell. Mol. Life Sci. 72(21), 4063-4076 (2015).

6 Warton K, Graham LJ, Yuwono N, Samimi G. Comparison of 4 commercial kits for the extraction of circulating DNA from plasma. Cancer Genet. 228-229, 143-150 (2018). 